\title{
Repercussões da deficiência de ferro durante a gestação e puerpério para o binômio materno-fetal
}

\author{
Repercussions of iron deficiency during pregnancy and pregnancy for the maternal-fetal \\ binomium
}
Repercusiones de la deficiencia de hierro durante el embarazo y el embarazo para el binomio materno-fetal

Vitoria Vilas Boas da Silva Bomfim ${ }^{1 *}$, Emilie dos Santos Aleluia', Tailine dos Santos Santana², Tainara Regina Castro Silva ${ }^{1}$, Leidiane Araújo Oliveira ${ }^{1}$, Adriele da Silva Brito ${ }^{1}$, Denise Mineiro Cunha Alves³, Nívea Maria Ferreira Gama¹, Êmile Tosta Araújo.

\section{RESUMO}

Objetivo: Analisar as repercussões da deficiência de ferro durante a gestação e no puerpério para o binômio materno-fetal, bem como destacar a intervenção do enfermeiro frente à problemática. Revisão bibliográfica: Deficiência de ferro em gestantes tem consequências relevantes que culminam na anemia gestacional prejudicando assim a saúde materna, acarretando prejuízos no desenvolvimento e crescimento fetal e podendo levar a perdas sanguíneas agudas no pós parto, levando a consequências irreversíveis para ambos, sendo assim, é necessário que haja uma identificação precoce da deficiência deste micronutriente na gestante, para que se possa montar estratégias de intervenção nutricional ou medicamentosa, promovendo níveis adequados dos mesmos, melhorando o prognóstico gravídico junto a gestante. $O$ enfermeiro é fundamental para o acompanhamento e desenvolvimento de uma gestação saudável, pois deve enfatizar, priorizar e orientar as recomendações passadas pelo médico, bem como reforçar e esclarecer sobre as boas práticas alimentares, e estilo de vida adequado, para assim evitar intercorrência e garantir uma gestação sem complicações. Considerações finais: A carência de ferro durante a gestação e puerpério para o binômio materno-fetal causa severas sequelas no desenvolvimento e crescimento fetal, além de prejudicar a saúde da mãe, podendo levar a consequências irreversíveis para ambos.

Palavras-chave: Anemia ferropriva, Gestantes, Puerperal, Anemia.

\section{ABSTRACT}

Objective: To analyze the repercussions of iron deficiency during pregnancy and in the puerperium for the maternal-fetal binomial, as well as to highlight the nurse's intervention in the face of the problem. Bibliographic review: Iron deficiency in pregnant women has relevant consequences that culminate in gestational anemia, thus impairing maternal health, causing losses in fetal development and growth and can lead to acute blood loss in the postpartum period, leading to irreversible consequences for both, thus, it is necessary to have an early identification of the deficiency of this micronutrient in the pregnant woman, so that nutritional or drug intervention strategies can be put together, promoting adequate levels of them, improving the pregnancy prognosis with the pregnant woman. The nurse is fundamental for the monitoring and development of a healthy pregnancy, as she must emphasize, prioritize and guide the recommendations given by the doctor, as well as reinforcing and clarifying about good eating practices, and an adequate lifestyle, in order to avoid complications and ensure a pregnancy without complications. Final considerations: Iron deficiency during pregnancy and the puerperium for the maternal-fetal binomial causes severe sequelae in fetal development and growth, in addition to harming the mother's health, which can lead to irreversible consequences for both.

Keywords: Iron deficiency anemia, Pregnant women, Puerperal, Anemia.

${ }^{1}$ Centro Universitário Jorge Amado (UNIJORGE), Salvador - BA.

*E-mail: mailto:vitoriavilasboas31@gmail.com

${ }^{2}$ Centro Universitário UNIFTC (UNIFTC), Salvador - BA.

${ }^{3}$ Hospital Aliança (HA), Salvador - BA.

SUBMETIDO EM: 9/2020

ACEITO EM: 10/2020

PUBLICADO EM: 12/2020

REAS/EJCH | Vol.12(12) | e5154 | DOI: https://doi.org/10.25248/reas.e5154.2020 Página 1 de 8 
RESUMEN

Objetivo: Analizar las repercusiones de la deficiencia de hierro durante el embarazo y en el puerperio para el binomio materno-fetal, así como destacar la intervención de la enfermera ante el problema. Revisión bibliográfica: La deficiencia de hierro en la gestante tiene consecuencias relevantes que culminan en anemia gestacional, perjudicando la salud materna, provocando pérdidas en el desarrollo y crecimiento fetal y puede conducir a una hemorragia aguda en el posparto, lo que conlleva consecuencias irreversibles para ambos, por lo que, es necesario tener una identificación precoz de la deficiencia de este micronutriente en la gestante, para que se puedan articular estrategias de intervención nutricional o farmacológica, promoviendo niveles adecuados de los mismos, mejorando el pronóstico del embarazo en la gestante. La enfermera es fundamental para el seguimiento y desarrollo de un embarazo saludable, ya que debe enfatizar, priorizar y orientar las recomendaciones dadas por el médico, así como reforzar y esclarecer sobre buenas prácticas alimentarias, y un estilo de vida adecuado, a fin de evitar complicaciones y asegurar un embarazo sin complicaciones. Consideraciones finales: La deficiencia de hierro durante el embarazo y el puerperio para el binomio materno-fetal ocasiona graves secuelas en el desarrollo y crecimiento fetal, además de perjudicar la salud de la madre, lo que puede tener consecuencias irreversibles para ambos.

Palabras clave: Anemia ferropénica, Gestante, Puerperal, Anemia.

\section{INTRODUÇÃO}

O ferro é um micronutriente e faz parte de inúmeras proteínas, incluindo as enzimas e a hemoglobina. A hemoglobina é fundamental no transporte do oxigênio para os tecidos e cerca de dois terços do ferro que está presente no corpo humano, encontra-se nos eritrócitos circulantes. A deficiência severa de ferro no organismo provoca a anemia por deficiência de ferro e acontece quando são esgotadas as reservas nutricionais de ferro, principalmente por razão do balanço negativo entre a ingestão e os requerimentos de ferro. Para evitar a deficiência recorrente deste micronutriente, é necessário que se avalie a ingestão alimentar seguida da orientação ao indivíduo, contribuindo assim com o tratamento e mudança de práticas alimentares (FERREIRA DSDS, et al., 2020).

Anemia é definida pela Organização Mundial de Saúde (OMS) como a condição na qual o conteúdo da hemoglobina no sangue está abaixo do normal, como resultado da carência de um ou mais nutrientes essenciais, podendo ser causada por inúmeros fatores, dentre os quais pode-se citar: deficiência nutricional de ferro, de zinco e de vitamina B12 (MINISTÉRIO DA SAÚDE, 2020).

Regularmente a anemia não é uma doença em si, mas sinaliza a existência de uma doença prévia que levou a instalação da anemia, por isso, quando um paciente apresenta tal síndrome não é necessário apenas que haja o tratamento pontual da anemia, mas que investigações mais profundas sejam realizadas a fim de que patologias mais graves, que estejam causando essa anemia, sejam descobertas e tratadas rapidamente (BAPTISTA LLS, 2019). Segundo a Organização Mundial de Saúde, o diagnóstico de anemia é definido por níveis de hemoglobina $(\mathrm{Hb})$ menores que $13 \mathrm{~g} / \mathrm{dL}$ para homens, $12 \mathrm{~g} / \mathrm{dL}$ para mulheres e $11 \mathrm{~g} / \mathrm{dL}$ para grávidas e crianças (WHO, 2019).

A carência de ferro causa a anemia ferropriva que é a anemia de maior prevalência encontrada no mundo e, até mesmo em países desenvolvidos, cerca de um terço dos pacientes a nível hospitalar são anêmicos. Esta síndrome clínica é detectada através de exames laboratoriais que mostram a diminuição na concentração de hemoglobina no sangue ou da concentração de hemácias por unidade de volume, em comparação com parâmetros do sangue periférico de uma população de referência (WALDIR C e MODOTTI $P, 2015)$. A anemia ferropriva pode ser ocasionada por inúmeros fatores que resultam da perda de ferro dentre eles: as hemorragias, as perdas urinárias, a ingestão ou absorção deficiente deste micronutriente (YAMAGISHI JA, et al., 2017).

A anemia é uma patologia prevalente em todo mundo, podendo afetar qualquer pessoa, independente de classe social, tendo em vista que as pessoas que possuem condições econômicas para manter uma nutrição 
adequada possuem deficiência de ferro assim como as pessoas que não possuem, mas aquelas em menor proporção. Vale ressaltar que apesar de poder afetar qualquer pessoa, existem grupos mais vulneráveis a desenvolverem anemia ferropriva destacando os lactentes, as gestantes, as crianças menores de cinco anos e as mulheres em idade fértil (FONSECA CEPS, et al., 2020).

Durante a gestação, a anemia, é considerada um problema de saúde global, podendo ser provocada por fatores fisiológicos, por deficiência de ferro ou outras causas menos comuns, e independente da causa, se não for tratada de forma adequada, irá ocasionar sérias consequências adversas para a saúde da genitora e do feto (AUERBACH M, et al., 2020).

Portanto, o acompanhamento médico e a realização do pré-natal é imprescindível, tendo em vista que, muitas vezes, as mulheres com anemia são assintomáticas, além disso, o diagnóstico precoce e 0 nivelamento do grau da anemia permite o tratamento rápido.

A avaliação das gestantes deve ser minuciosa, levando em conta uma anamnese detalhada e um exame físico completo, já que nos dois primeiros semestres o Volume Corpuscular Médio (VCM) das grávidas aumentam discretamente, podendo mascarar uma anemia ferropriva. O melhor teste laboratorial para avaliar a quantidade de ferro disponível é a dosagem da ferritina sérica, a qual está diretamente relacionada aos depósitos de ferro (REZENDE J e MONTENEGRO CA, 2018).

No período correspondente ao puerpério, tempo de seis a oito semanas após o parto, o corpo da parturiente também passa por intensas modificações e transformações anatômicas e de adaptação psicoorgânicas, no qual ocorre o processo de involução de órgãos reprodutivos à situação pré-gravídica, o estabelecimento da lactação e a ocorrência de intensas alterações emocionais e hormonais.

Essas mudanças são involuntárias e provoca, sérios desequilíbrios no estado de humor da puérpera, deixando-as mais sensíveis e vulneráveis. A deficiência de ferro no pós parto atinge a relação mãe e filho, provocando fadiga e a exaustão da mãe, levando a uma grande influência na produção e qualidade do leite, possibilitando baixo peso ao recém-nascido (RN) (ANDRADE RD, et al., 2015).

Aproximadamente $40 \%$ das morte maternas e perinatais são ligadas à anemia, o que revela a importância dessa discussão para que políticas públicas de saúde voltadas para a prevenção da anemia e acompanhamento dessas gestantes sejam efetivas reduzindo o número de óbitos e sequelas para mãe e fetos (WHO, 2018).

Diante do exposto, é evidente que a deficiência deste micronutriente traz sequelas a curto e longo prazo para o indivíduo, principalmente em gestantes cujo corpo sofre alterações fisiológicas. Nesta perspectiva, este estudo tem como objetivo, analisar as repercussões da deficiência de ferro durante a gestação e o puerpério para o binômio materno-fetal, bem como destacar a intervenção do enfermeiro frente à problemática.

\section{REVISÃO BIBLIOGRÁFICA}

\section{Repercussões da deficiência de ferro durante a gestação para o binômio materno-fetal}

O ferro é um nutriente extremamente importante para a saúde do indivíduo, pois a sua carência pode desencadear uma desordem nutricional (CARDOSO MA, 2013). Este mineral desempenha uma importante função no metabolismo do organismo, tais como transporte e armazenamento de oxigênio, síntese do DNA, co-fatores de algumas reações enzimáticas entre outras, e a sua ingestão adequada é fundamental para que organismo funcione normal (SOARES CFT, et al., 2019).

É necessário o equilíbrio do ferro no corpo, pois sua carência pode levar a um distúrbio no seu processo farmacocinético resultando em uma deficiência que pode interferir nas funções vitais e levar ao óbito (TEODORO L, et al., 2016). A anemia, consequência da deficiência de ferro, é um distúrbio nutricional grave, de grande relevância mundial e é um problema de saúde pública afetando tanto países desenvolvidos quanto subdesenvolvidos (ZUFFO CRK, et al., 2016).

Deficiência de ferro quando refere-se às gestantes tem consequências relevantes que culminam na anemia gestacional prejudicando assim a saúde materna, acarretando prejuízos no desenvolvimento e crescimento fetal e podendo levar a perdas sanguíneas agudas no pós parto, levando a consequências 
irreversíveis para ambos, sendo assim, é necessário que haja uma identificação precoce da deficiência deste micronutriente na gestante, para que se possa montar estratégias de intervenção nutricional ou medicamentosa, promovendo níveis adequados dos mesmos, melhorando o prognóstico gravídico junto a gestante (CARDOSO MA, 2013).

Presume-se que $30 \%$ das mulheres em idade reprodutiva são anêmicas e segundo a OMS, a prevalência é ainda maior em gestantes, estimando-se que mais de $40 \%$ das mulheres grávidas em todo o mundo têm anemia, podendo existir alternâncias nas prevalências regionais e globais de anemia durante a gravidez a partir do status socioeconômico e as deficiências nutricionais associadas (AUERBACH M, et al., 2020).

Estimativas mostram que mais da metade das mulheres grávidas em todo mundo apresentam anemia ferropriva, $52 \%$ delas encontradas em países não industrializados e $23 \%$ em países industrializado. Estudos revelam que nas mulheres afro-americanas, a prevalência de anemia durante a gravidez é de $27 \%$ enquanto que entre as mulheres brancas não hispânicas é de 7\% (WALDIR C e MODOTTI P, 2015; AUERBACH M, et al., 2020).

As causas mais comuns de anemia em mulheres grávidas são a anemia fisiológica da gravidez e a deficiência de ferro e essas duas condições são os agentes causadores, na maioria das vezes, da baixa concentração de hemoglobina durante a gravidez. No entanto, outras causas responsáveis por provocar anemia em gestantes, não devem ser negligenciadas (AUERBACH M, et al., 2020).

Durante a gravidez, ocorre o aumento do volume sanguíneo na mulher por ação dos hormônios estrogênio e progesterona, que sofrem influência do sistema renina angiotensina aldosterona, a fim de atender adequadamente às demandas da gestante e do feto (WHO, 2019).

Esta elevação da volemia não é acompanhada do aumento dos eritrócitos e isto ocorre em resposta da eritropoietina que estimula a eritropoiese medular. Assim, ocorre diminuição da resistência vascular periférica para acomodação desta expansão volumétrica (MENDONÇA IMC, et al., 2020; DARNTON -HILL e MKPARU UC, 2015).

Por outro lado, o corpo da mulher sofre alterações fisiológicas e o organismo aumenta a necessidade de ferro para prover a expansão da massa eritrocitária evitando assim a redução do volume sanguíneo da própria gestante e que será imprescindível para a formação do sangue da placenta e do feto (PESSOA LS, et al., 2015; MINISTÉRIO DA SAÚDE, 2000; PAULA WKA, et al., 2016). As adaptações gravídicas são possíveis por causa do aumento da taxa de absorção do ferro, mas também devido a elevação da transferrina circulate estimulada pelo estrogênio.

Todavia, mesmo existindo todos esses mecanismos compensatórios, o aumento do volume eritrocitário não acompanha o aumento do volume plasmático, consequentemente acontece uma queda do hematócrito, hemoglobina e viscosidade sanguínea, levando a um estado de hemodiluição (anemia fisiológica da gravidez) (RODRIGUES LP e JORGE SRPF, 2010). No ponto de comprometimento fetal, a deficiência de ferro pode levar ao quadro de anemia e provocar perdas gestacionais, como abortamento ou óbitos uterinos, prematuridade, hipoxemia fetal, ruptura prematura das membranas ovulares, levando ao quadro de infecção, restrição do crescimento fetal e algumas vezes comprometimento irreversível no desenvolvimento neurológico fetal (PAULA WKA, et al., 2016).

Estudos evidenciam que o micronutriente ferro auxilia no processo de formação da hemoglobina fetal e também no desenvolvimento do sistema nervoso central, agindo através da síntese de enzimas responsáveis pelo metabolismo cerebral, sua deficiência promoveria problemas na formação e desenvolvimento deste feto. $E$ ao nascimento, os bebês nascidos de mães com deficiência de ferro, correm alto risco de apresentar este mesmo problema, levando a um quadro de anemia neonatal (FISBERG M, et al., 2018; AUERBACH M, et al., 2020).

A insuficiência do consumo do ferro durante a gestação pode afetar o desenvolvimento do cérebro no recém-nascido, resultando a sequelas no desenvolvimento físico e mental, como diminuição da capacidade cognitiva, aprendizagem, concentração, memorização e alteração do estado emocional. Estudos revelam que não há uma informação exata sobre o mecanismo de comprometimento, entretanto, sua carência contribui 
para as alterações no metabolismo de neurotransmissores como também na constituição da bainha de mielina (GARDNER $\mathrm{H}$, et al., 2018).

Nem sempre o consumo normal de ferro é suficiente para preencher tal necessidade, até mesmo para compensar a perda durante o parto, por isso, faz-se necessário uma nutrição adequada, com oferta apropriada de ferro e a suplementação deste micronutriente durante todo o período gestacional, uma vez que sua deficiência pode significar maiores riscos de parto prematuro e até mesmo aumento da morbimortalidade materna pós parto (PAULA WKA, et al., 2016; KREBS N, et al., 2016).

Visto que a saúde da mãe e do feto pode ser afetada por anemia durante a gravidez, a sua identificação precoce, a prevenção e o tratamento o quanto antes é provavelmente benéfica para ambos. A promoção da correção da deficiência de ferro antes do terceiro trimestre é ideal, pois a neurogênese dependente de ferro é máxima durante o terceiro trimestre e sua deficiência durante esse período tem sido associada a déficits no desenvolvimento neurocognitivo. Por isso faz-se necessário o acompanhamento mensal desta gestante com intuito de detectar e resolver o mais rápido possível os casos de anemia por deficiência de ferro (AUERBACH M, et al., 2020).

O tratamento da deficiência de ferro iniciado após o diagnóstico de anemia por deficiência de ferro pode ser tarde demais para evitar alguns resultados adversos portanto há indicação de suplementação de ácido fólico pré concepcional e até a $12^{\circ}$ semana de gestação com intuito de prevenir defeitos na formação do tubo neural fetal. Para o tratamento é indicado a ingestão oral de sulfato ferroso que deve continuar após 3 meses da normalização da Hb e no mínimo 6 semanas após o parto.

Esta terapêutica é eficaz para a maioria das mulheres, além de ser segura, barata e prontamente disponível. E nos casos de anemia moderada a grave, falha de resposta ou contraindicação para ferro oral, intolerância ao ferro oral, má absorção ou necessidade de reposição rápida a indicação é administração de ferro via endovenosa. A transfusão sanguínea só é indicada para aquelas que apresentam sintomas significativos associados à anemia grave ou para os quais a transfusão é indicada por outros motivos (FERREIRA LB, et al., 2018).

\section{Consequências da carência do micronutriente no período puerperal}

Dentre as consequências da anemia no período gestacional, é visto uma maior ocorrência de sangramentos durante $o$ parto e no puerpério, resultando no aumento da morbidade e mortalidade maternal (AREIA AL, et al., 2019). Estudos mostram que a prevalência da anemia pós-parto pode ser comum variando de 22 a $29 \%$ e pode estar associado a parto instrumental e remoção manual de placenta em 35 e $60 \%$ dos casos e se a anemia é provocada por hemodiluição devido à fisiologia da gravidez ou por perda de sangue sem deficiência de ferro, é provável que o problema se resolva dentro de algumas semanas (AUERBACH M, et al., 2020).

No período puerperal imediato ocorre a loquiação, condição fisiológica do parto normal, e que diminui conforme a redução da contratilidade uterina, assim como a cicatrização dos tecidos do canal vaginal lacerados durante o nascimento, caso isto não ocorra normalmente é necessário a suplementação do ferro (SAUNDERS C, et al., 2016). Deve haver muito cuidado em casos onde a loquiação deixa de ser fisiológica e passa a ser patológica, devido a condições de atonia uterina, levando a choque hipovolêmico e morte materna. Quando isto ocorre, o tratamento para o choque deve ser instaurado de maneira imediata a fim de evitar mortalidade materna bem como a correção da anemia grave (PINHO JG e BUCHNER G, 2020).

Neste período observa-se também o aumento da probabilidade da parturiente desenvolver uma depressão pós-parto, fadiga, palidez, náusea, tontura, palpitações, diminuição do apetite comprometendo o sistema imune e consequentemente redução da capacidade de combater infecções, causar sentimentos de tensão, ansiedade, síndrome do leite insuficiente e redução da qualidade do leite, nesses casos o ideal é abrir mão de testes laboratoriais, como um hemograma completo, para avaliar presença de anemia e poder tratá-la (AUERBACH M, et al., 2020; ALMEIDA NMC e ARRAIS AR, 2016).

Identificado a anemia, serão estabelecidas as medidas de combate à deficiência de ferro e à anemia ferropriva, consistindo de forma resumida a modificação de hábitos alimentares, os tornando mais saudáveis; 
diagnosticando e tratando dos fatores de perda crônica de sangue; controlando as infecções e infestações que colaboram para geração e agravo da anemia; fortalecimento de alimentos e uso de suplementação medicamentosa com base de sais de ferro (SAUNDERS C, et al., 2016).

\section{Intervenção do enfermeiro frente à problemática}

Na saúde da família o enfermeiro desenvolve seu trabalho em dois campos: na Unidade Básica de Saúde (UBS) e na comunidade, a fim de apoiar e supervisionar o trabalho do agente comunitário de saúde (ACS) e do auxiliar de enfermagem, tal como dando assistência às pessoas que tem a necessidade de um atendimento domiciliar. Dependendo do cargo que ocupa na saúde da família, o profissional de enfermagem desenvolve diversas atividades, sejam elas em maior ou menor complexidade. O enfermeiro tem a capacidade de desenvolver atividades para aperfeiçoamento pessoal, e através de seu conhecimento técnico-científico, garantir a manutenção de condições para prestar um atendimento eficiente e humanizado para a pessoa assistida (BATISTA AP, et al., 2020).

O pré-natal configura-se como um momento importante de promoção à saúde da gestante e do feto e é considerado fundamental na prevenção e/ou detecção precoce de patologias tanto maternas como fetais, permitindo um desenvolvimento saudável do bebê e reduzindo as taxas de morbimortalidade materna e infantil, adotando-se medidas que assegurem a melhoria do acesso, da cobertura e da qualidade do acompanhamento ao pré-natal, da assistência ao parto e puerpério e assistência neonatal (FERREIRA LB, et al., 2018; CAMPAGNOLI M, et al., 2020).

O acompanhamento de pré-natal é de extrema importância no período gestatório e é bastante relevante as ações do enfermeiro nesta assistência, cabendo-Ihe prestar um atendimento qualificado de acordo com as necessidades da gestante (LEMES AG, 2012). O plano de cuidados de enfermagem para a gestante com anemia ferropriva deve ser realizado de forma individualizada, baseando-se nos diagnósticos de enfermagem encontrados e com os seguintes objetivos: prevenir as complicações relacionadas à anemia ferropriva, para que os valores de hemoglobina voltem aos níveis normais; crescimento e desenvolvimento fetal adequado e que não apresente as alterações relacionadas a anemia materna e a realização do tratamento de forma eficaz e assertiva (PINHO JG; BUCHNER G, 2020).

Este cuidado deve ser iniciado a partir da prevenção e continuado até o tratamento da anemia tendo como finalidade essencial do cuidado de enfermagem a orientação sobre alimentação adequada (RODRIGUES LP e JORGE SRPF, 2010). A gestante tem que ser orientada sobre as dietas variadas que proporcionam a ingestão e absorção adequada do micronutriente (ferro) e qual a importância da suplementação férrica.

As orientações de enfermagem são muito importantes no cuidado pré-natal, pois auxiliam o enfermeiro a planejar a assistência individualizada destacando os pontos relevantes da alimentação. Os protocolos de orientação de enfermagem focado na gestante com anemia ferropriva, garantem melhores efeitos na prevenção e no tratamento desta patologia (RODRIGUES LP e JORGE SRPF, 2010).

Os profissionais de enfermagem são de suma importância nesse momento pois os mesmos orientam as gestantes, sanando suas dúvidas e orientando-as quanto a importância das consultas de pré natal, porque por meio dessas consultas é que se identifica as intercorrências precocemente e monitora as gestantes que se encontram em situações de riscos. As gestantes que possuem alteração nos exames hematológicos são acompanhadas atentamente, caso o resultado seja muito grave as mesmas são encaminhadas para consultas com obstetras (LEMES AG, 2012).

Os principais diagnósticos seguindo a taxonomia NANDA para estas gestantes são: Nutrição inadequada: ingesta menor que as necessidades corporais, relacionada a verbalização de falta de alimento, Nutrição desequilibrada: ingesta maior que as necessidades corporais, relacionada a falta de conhecimento nutricional básico; Risco para infecção relacionado a defesa secundária insuficiente pela diminuição da hemoglobina; Manutenção do lar prejudicada relacionada a falta de recursos financeiros suficientes; Déficit de conhecimento sobre a alimentação adequada durante a gestação; Risco para lesão fetal relacionado a diminuição da perfusão uteroplacentária e Risco para não comprometimento relacionado a dieta alimentar recomendada para o tratamento (BARROS SMO e COSTA CAR, 2011). 
O enfermeiro da estratégia de saúde da família tem como função orientar as mulheres da comunidade e usuárias do serviço de saúde local a iniciar o planejamento familiar e/ou pré natal assim que descobre a gestação. É através da anamnese e exame físico que se constata diagnósticos e planeja a melhor intervenção a se fazer, se é necessário um planejamento adequado sobre sua ingesta alimentar, dentro da realidade do paciente, bem como prescrever e fornecer todas as orientações sobre o uso do suplemento alimentar, que irá prevenir eventual intercorrência antes, durante e após a gestação (TAVARES DS, et al., 2019).

O mesmo, também pode prescrever suplementação de ferro e ácido fólico desde o momento que a gestante decidir engravidar $40 \mathrm{mg}$ de ferro elementar e 400 microgramas de ácido fólico diariamente até o final da gestação. No pós parto e pós-aborto é indicado o uso de $40 \mathrm{mg}$ de ferro elementar até o terceiro mês após o evento (NUTES, 2020).

Estudos evidenciam que o sulfato ferroso, complemento vitamínico alimentar, é um suplemento fundamental na prevenção de doenças carenciais, na formação de tecidos essenciais na gestação, pois ocorrerá um aumento de produção de hemácias maternal e nos tecidos que vão constituir o feto (BARCO BT, et al., 2015).

Vale ressaltar que o papel do enfermeiro é fundamental para o acompanhamento e desenvolvimento de uma gestação saudável, pois deve enfatizar, priorizar e orientar as recomendações passadas pelo médico, bem como reforçar e esclarecer sobre as boas práticas alimentares, e estilo de vida adequado, para assim evitar intercorrência e garantir uma gestação sem complicações.

\section{CONSIDERAÇÕES FINAIS}

O período gestacional envolve diversas mudanças fisiológicas para assegurar o crescimento e desenvolvimento fetal. A carência de ferro durante a gestação para o binômio materno-fetal causa severas sequelas no desenvolvimento e crescimento fetal, além de prejudicar a saúde da mãe, podendo levar a consequências irreversíveis para ambos. As repercussões associada a deficiência do micronutriente para o organismo, leva a desfechos desfavoráveis na gestação e no período puerperal podendo levar a morbimortalidade de ambos. É de suma importância o acompanhamento dessas gestantes para tentar suprir e corrigir a carência do mineral, sendo o pré natal um instrumento imprescindível e importante, voltado para o acompanhamento e avaliação da gestante, com intuito de viabilizar uma gestação saudável e segura com menos riscos de complicações para o feto e mãe, garantindo estratégias de promoção e prevenção de saúde. Neste contexto destaca-se o papel fundamental da enfermagem, pois é quem faz o acompanhamento das gestantes no pré-natal e no período puerperal, dando todo suporte e orientações adequadas.

\section{REFERÊNCIAS}

1. ALMEIDA NMC, ARRAIS AR. O Pré-Natal Psicológico como Programa de Prevenção à Depressão Pós-Parto. Psicol. cienc. prof. [online]. 2016; 36(4): 847-863.

2. ANDRADE RD, et al. Fatores relacionados à saúde da mulher no puerpério e repercussões na saúde da criança. Esc. Anna Nery, Rio de Janeiro, 2015; 19(1): 181-186.

3. AREIA AL, et al. Anemia na gravidez e no puerpério Normas de Orientação da SPOMMF. Acta Obstet Ginecol Port, 2019; 13(2).

4. AUERBACH M, et al. Anemia in pregnancy. UpToDate. 2020.

5. BAPTISTA LLS. Prática alimentar e determinantes sociais na anemia em famílias atendidas pela Estratégia Saúde da Família no estado do Maranhão - estudo de base populacional [thesis]. São Paulo, Faculdade de Saúde Pública; 2019.

6. BARCO BT, et al. Knowledge of supplemental folic acid during pregnancy. Invest. educ. enferm [online]. 2015; 33(3): 456-464.

7. BARROS SMO, COSTA CAR. Consulta de enfermagem a gestantes com anemia ferropriva. Rev. Latino-Am. Enfermagem [online]. 2011; 7(4): 05-111.

8. BATISTA AP, et al. O Enfermeiro Como Supervisor e Educador do Agente Comunitário de Saúde. Revista de Trabalhos Acadêmicos-Universo Juiz De Fora, 2018.

9. BREYMANN C, AUERBACH M. Iron deficiency in gynecology and obstetrics: clinical implications and management. Hematology Am Soc Hematol Educ Program 2017; 2017 (1): 152-159.

10. CAMPAGNOLI M, et al. Atendimento de pré natal na estratégia saúde da família: a singularidade da assistência de enfermagem. 2020.

11. CARDOSO MA. Nutrição e Metabolismo: Nutrição Humana. 1. Ed. Rio de Janeiro: Guanabara Koogan, 2013. 
12. CHUA S, et al. Intravenous iron vs blood for acute post-partum anaemia (IIBAPPA): a prospective randomized trial. BMC Pregnancy Childbirth, 2017; 17: 424.

13. DARNTON-HILL I, MKPARU UC. Micronutrients in Pregnancy in Low- and Middle-Income Countries. Nutrients, 2015; 7: $1744-1768$.

14. FERREIRA DSDS, et al. Consumo alimentar de ferro e cálcio por adolescentes em fase reprodutiva de uma escola pública de Petrolina-Pernambuco. Revista de Atenção à Saúde. 2015: 13(45): 49-54.

15. FERREIRA LB, et al. Fatores assistenciais e gestacionais associados à anemia em nutrizes atendidas em um banco de leite humano. Ciênc. saúde colet. 2018: 23(11).

16. FISBERG M, et al. Consenso Sobre Anemia Ferropriva: Mais que uma Doença, uma Urgência Médica! Sociedade Brasileira de Pediatria, 2018;2.

17. FONSECA CEPS, et al. Educação alimentar e suplementação de micronutrientes: a visão de enfermeiras integrantes do programa saúde na escola e do programa nutrisus. V Congresso em Desenvolvimento Social, 2016.

18. GARDNER $\mathrm{H}$, et al. Observations on the health of infants at a time of rapid societal change: a longitudinal study from birth to fifteen months in Abu Dhabi. BMC Pediatr, 2018; 18(32).

19. KREBS N, et al. Neurodevelopment: The Impact of Nutrition and Inflammation During Infancy in Low Resource Settings. Pediatrics. [Online], 2016.

20. LEMES AG. Assistência de enfermagem à gestante na primeira consulta de pré-natal. Revista Eletrônica da Univar, 2012; 1(8): 70-73.

21. MENDONÇA IMCC, ESTEVÃO MLF. Hematologia da Gravidez 2015. Universidade de Lisboa. Tese de Doutorado.

22. MINISTÉRIO DA SAÚDE. Secretaria de Atenção à Saúde Departamento de Atenção Básica B. Gestação de Alto Risco: Manual Técnico [Internet], 2000; 105-107.

23. NÚCLEO DE TELESSAÚDE - NUTES PE. Como utilizar o ácido fólico no período gestacional? [cited 2020 May 19].

24. PAULA WKA, et al. Prevalência de Anemia em Gestantes Acompanhadas nas Unidades Básicas de Saúde do Município de Caruaru-PE. 2016.

25. PESSOA LS, et al. Evolução temporal da prevalência de anemia em adolescentes grávidas de uma maternidade pública do Rio de Janeiro. Rev. Bras. Ginecol. Obstet. [Internet]. 2015; 37(5): 208-215.

26. PINHO JG, BUCHNER G. Prevalência e fatores associados à Anemia no Pós-Parto recente no Centro Materno-Infantil do Norte, 2020.

27. REZENDE J, MONTENEGRO CA. Obstetrícia Fundamental -14 a Edição - Saraiva [Internet]. 2018

28. RODRIGUES LP, JORGE SRPF. Deficiência de ferro na gestação, parto e puerpério. Rev. Bras. Hematol. Hemoter. [Internet], 2010; 32(2): 53-56.

29. SAUNDERS C, et al. Determinants of gestational night blindness in pregnant women from Rio de Janeiro, Brazil. Public Health Nutrition. [Online] Cambridge University Press; 2016 ;19(5): 851-860.

30. SOARES CFT, et al. Composição centesimal e análise do teor de ferro nas farinhas de trigo fortificadas comercializadas nos municípios de governador valadares e lavras - Minas Gerais. 2019.

31. TAVARES DS, et al. Sistematização da assistência de enfermagem no pré-natal: revisão integrativa. REAS [Internet], 2019; (31): 1255.

32. TEODORO L, et al. Prevalence and risk factors of anemia in children. Jornal de Pediatria (Versão em Português), 2016; 92(4)

33. WALDIR C, MODOTTI P. Revisão Medicina (Ribeirão Preto), 2015; 48(4): 401-407.

34. WHO | Archived: Iron deficiency anaemia: assessment, prevention and control. WHO. 2018.

35. WHO | Vitamin and mineral requirements in human nutrition. WHO. 2019.

36. YAMAGISHI JA, et al. Anemia ferropriva. Rev Cient Fac Educ e Meio Ambient [Internet], 2017.

37. ZUFFO CRK, et al. Prevalence and risk factors of anemia in children. Jornal de Pediatria (Versão em Português), 2016; 92(4) 353-360. 